\title{
Ewa Urbaniak*
}

\section{La traduzione delle espressioni deittiche}

\section{La definizione della deissi}

Ogni lingua costituisce un sistema degli elementi la cui funzione si radica nell'atto di descrivere la nostra percezione del mondo. Nel sistema linguistico possiamo trovare degli elementi il cui significato si riferisce ai concetti universali e comprensibili per tutti i parlanti. Tale è il caso di un'ampia gamma di espressioni che sembrano intelligibili anche fuori dal loro contesto pragmatico.

D'altra parte, non tutte le espressioni linguistiche diventano così facili da identificare. Che significa, ad esempio, la parola qui? Con l'obiettivo di comprovarlo, analizziamo le seguenti conversazioni':

(1) - Come ha conosciuto l'AGOP e perché dedica una parte del suo tempo a questa Associazione?

- Io non ne parlo mai con nessuno ma mi trovo qui, in questa associazione, da genitore.

(2) - Raccontaci del tuo amore per il rugby, e delle analogie e delle differenze nell'essenza di questi due sport.

- Il calcio, a me, in particolare qui in Italia, crea un certo fastidio.

Nel primo caso l'avverbio qui equivale all'Associazione AGOP, mentre nel secondo, all'Italia. Come possiamo osservare, lo stesso elemento assume un significato totalmente diverso a seconda della situazione comunicativa. Siccome non esiste un concetto prototipico che determini la nostra visione del qui, in ogni enunciato si possono manifestare dei valori diversi.

* Uniwersytet Łódzki, e-mail: ewa.urbaniakowa@gmail.com

1 Tutti gli esempi italiani nell'articolo provengono da: https://www.unistrapg.it/cqpweb/pec (23/04/2016). 
Un caso particolare delle unità il cui significato dipende dalla situazione comunicativa costituiscono le espressioni deittiche, profondamente studiate dai numerosi linguisti (Bühler [1934] 2004; Coseriu 1989; Fillmore 1997; Vicente Mateu 1994; Vanelli e Renzi 1995). Sono tutti gli elementi la cui funzione si radica nel segnalare la localizzazione degli oggetti nello spazio oppure nel tempo (Carbonero Cano 1979: 13). In altre parole, grazie alla deissi i parlanti hanno la possibilità di delimitare l'ubicazione di una cosa o il tempo nel quale si svolge un'azione. Negli esempi (1) e (2) il deittico qui dimostra dove si trovano i soggetti delle frasi: marca la loro localizzazione nello spazio.

Per delimitare la posizione di certi oggetti, gli elementi deittici richiedono un punto di riferimento, ossia un posto secondo il quale si può determinare la loro localizzazione. Ogni lingua presenta le differenti dimensioni che determinano i punti di riferimento (Ruiz Antón 1998: 44). In alcune lingue, il punto di riferimento dipende dal movimento o dalla localizzazione di un fiume. Ciò nonostante, nella nostra percezione delle lingue, basata sulla visione eurocentrica, i punti di riferimento della deissi corrispondono alla situazione spazio-temporale delle persone coinvolte (o meno) nella conversazione. In altre parole, il parlante segnala gli oggetti rispetto all'ubicazione delle persone diverse. Ci sono tre figure che si possono tener presenti:

a) l'emittente: la persona che pronuncia l'enunciato, corrisponde all'IO,

b) l'ascoltatore: la persona a cui si rivolge l'enunciato, corrisponde al TU,

c) la persona non presente nella conversazione (non tutte le lingue la codificano come punto di riferimento): corrisponde al LUI.

Secondo la divisione menzionata, un oggetto si può trovare:

a) vicino all'emittente;

b) vicino all'ascoltatore;

c) vicino alla terza persona, vuol dire, lontano tanto dall'emittente, quanto dall'ascoltatore.

La ripartizione delle funzioni deittiche riguarda la localizzazione tanto nello spazio, quanto nel tempo. Per poter rendersi conto del funzionamento delle unità deittiche, analizziamo i seguenti esempi:

(3) Sì E ora ti do quest' altro pezzo di creta e mi fai vedere con questo martello come ti senti quando non puoi dire qualcosa Mi sento frustrato Ecco, puoi farmi vedere con il martello come è essere frustrato?

(4) ci va quotidianamente quasi a portare i soldi ricavati e poi risponde di tutto quanto quello che succede in quella zona è lui che organizza le estorsioni le rapine i sequestri eccetera eccetera

(5) No, no, questa settimana no, sto preparando le partite di domenica.

(6) in quella settimana in cui si verificava, si svolgeva il vertice lì a Genova. 
Gli esempi (3) e (4) concernono la delimitazione della situazione spaziale. Questo è un aggettivo dimostrativo che indica la vicinanza rispetto all'emittente. Nell'esempio (3) si caratterizza al martello con il dimostrativo questo, e così vi si rende conto di quello che l'oggetto in questione si trova vicino all'emittente. Nell'esempio (4), d'altra parte, si rammenta una zona lontana tanto dall'emittente, quanto dall'ascoltatore. Per questo motivo, si applica l'altro dimostrativo quello la cui funzione viene delimitata come il marcare della lontananza rispetto alle persone presenti nella conversazione.

Gli esempi (5) e (6) dimostrano come funzionano le unità deittiche rispetto alla situazione temporale. In caso del (5), il parlante considera la settimana un momento prossimo, per questo applica il dimostrativo questa per caratterizzarla. Nell'esempio (6) la settimana di cui si parla viene reputata remota: l'emittente utilizza il dimostrativo quello per dimostrare che costituisce un momento lontano dal tempo presente.

Come possiamo osservare, i deittici sono il modo più economico di delimitare la posizione spazio-temporale degli oggetti (Moreno Cabrera 1987: 75-76). In altre parole, un'unità semplice come questo può riferirsi ad un'ampia gamma di luoghi. Così, nell'esempio (3), invece di dire il martello che si trova vicino a me basta applicare il dimostrativo questo, che è capace di trasmettere lo stesso valore significativo.

\section{Le unità deittiche in italiano, spagnolo e polacco}

L'obiettivo della mia analisi è la delimitazione dei possibili problemi che si possono incontrare/manifestare traducendo gli elementi deittici. Le lingue che esaminiamo sono l'italiano, lo spagnolo e il polacco. Sebbene tutte le lingue in questione appartengano alla famiglia indoeuropea (e, per questo, condividono alcune caratteristiche), si possono osservare varie differenze tra i loro sistemi del segnalamento dello spazio e del tempo.

Nella mia analisi prendo in considerazione tre categorie grammaticali che vengono considerate come le possibili unità deittiche: gli aggettivi dimostrativi, gli avverbi dimostrativi ed alcuni verbi (Vanelli e Renzi 2001: 269). I diversi sistemi deittici vengono presentati nelle seguenti tabelle:

\begin{tabular}{|c|c|c|c|}
\hline & Italiano & Spagnolo & Polacco \\
\hline Emittente (io) & questo & este & ten \\
\hline Ascoltatore (tu) & $\begin{array}{c}\text { (codesto: solo in alcuni } \\
\text { dialetti) }\end{array}$ & ese & - \\
\hline $\begin{array}{c}\text { La persona non presente } \\
\text { (lui) }\end{array}$ & quello & aquel & tamten \\
\hline
\end{tabular}

AGGETTIVI DIMOSTRATIVI 


\begin{tabular}{|c|c|c|c|}
\hline & Italiano & Spagnolo & Polacco \\
\hline Emittente (io) & qui/qua & aquí (o acá) & tutaj \\
\hline Ascoltatore (tu) & $\begin{array}{c}\text { (costi/costà: solo in } \\
\text { alcuni dialetti) }\end{array}$ & ahí & tam \\
\hline $\begin{array}{c}\text { La persona non } \\
\text { presente (lui) }\end{array}$ & lì/là & allí (o allá) & \\
\hline
\end{tabular}

AVVERBI DIMOSTRATIVI

\begin{tabular}{|c|c|c|c|}
\hline & Italiano & Spagnolo & Polacco \\
\hline Emittente (io) & venire & venir & \multirow{2}{*}{ iść/przyjść } \\
\hline Ascoltatore (tu) & venire & ir & \\
\hline $\begin{array}{c}\text { La persona non } \\
\text { presente (lui) }\end{array}$ & andare & ir & \\
\hline
\end{tabular}

VERBI

\section{I problemi principali nella traduzione degli elementi deittici}

Sebbene i sistemi deittici delle lingue in questione si possano percepire simili, la loro traduzione rivela una serie di problemi che costituiscono grandi ostacoli per i traduttori.

\subsection{La percezione dello spazio}

Lo spazio costituisce un fenomeno esaminato dal punto di vista di varie discipline (Brzozowska-Zburzyńska 2012: 93-98). In fisica, lo spazio è una categoria obiettivamente misurabile (nei centimetri, metri, chilometri, ecc.). Lo spazio concettuale, d'altra parte, equivale alla nostra percezione della distanza tra diversi oggetti. Questa percezione è totalmente soggettiva e varia a seconda della persona e della sua esperienza.

Le nozioni più ovvie costituiscono lo spazio del parlante e lo spazio della terza persona (è facile da differenziare tra la zona dove si svolge la conversazione e, in conseguenza, la zona dove non si incontrano gli interlocutori). È la separazione tra lo spazio dell'emittente e quello del ricevente/destinatario che sembra meno chiara. Se due persone si trovano una di fianco all'altra, come possiamo verificare qual è la zona di una persona, e qual è la zona dell'altra? Tutto dipende dalla percezione dello spazio per gli interlocutori che la codificano tramite gli elementi deittici. 
(7) - Ma tu sei qui e ogni giorno è un giorno di festa.

(8) - Non hai paura, cioè non so, sei lì da sola?

- No, per niente.

Nella frase (7) il parlante usa l'avverbio qui che indica la zona dell'emittente dell'enunciato. Questo significa che il parlante percepisce che l'ascoltatore condivide con quest'ultimo la stessa area. Nel caso del (8), appare l'avverbio li vincolato alla zona lontana dalla figura del parlante. In questo modo, il parlante sottolinea che c'è un confine tra il suo spazio e lo spazio del suo interlocutore.

La questione di come percepire lo spazio si può osservare in tutte e tre le lingue che costituiscono l'obiettivo della mia analisi. Ciò nonostante, ogni idioma gode di una struttura distinta delle unità deittiche e, per questo motivo, le stesse nozioni vengono rappresentate tramite diversi mezzi linguistici.

\subsection{La figura dell'ascoltatore}

Come dimostrano le tabelle, la figura dell'ascoltatore non è sempre presente nella coscienza del parlante. In altre parole, non tutte le lingue codificano la vicinanza di un'entità al ricevente dell'enunciato. Lo spagnolo comprende la figura dell'ascoltatore nel suo sistema degli aggettivi e degli avverbi dimostrativi: le forme ese e ahí. Per quanto riguarda l'italiano, esistono le forme codesto e costì/costà, ma solo in alcuni dialetti (Ledgeway 2015: 63-80). D’altra parte, nel sistema verbale italiano esiste l'espressione venire che può indicare un movimento verso il ricevente dell'enunciato. In polacco non c'è alcuna forma che possa localizzare un ente rispetto all'ascoltatore.

Per visualizzare la problematica esposta, prendiamo come esempio la seguente frase:

(9) Como... como te digo, mirá [sic], recién compré la casa, donde esta la tienda, compré todo el edificio, sí. Enc. - ¿Esa casa no era de ustedes? Inf.b. - No, nunca ha sido nuestra, recién la compré. ${ }^{2}$

La traduzione del frammento che comprende l'espressione esa casa non è così facile se prendiamo in considerazione la mancanza di un elemento corrispondente nell'italiano standard (che non include la forma codesto) e nel polacco. Crediamo che ci siano due possibilità delle traduzioni:

a) l'omissione dell'allusione alla figura dell'ascoltatore:

la traduzione italiana: Questa casa non era loro/vostra?3

la traduzione polacca: Ten dom nie należat do państwa?

2 Tutti gli esempi spagnoli provengono da: http://www.corpusdelespanol.org/x.asp (24/04/2016).

3 II pronome loro indica la forma di cortesia. 
In questo caso, l'omissione suppone che questa in italiano e ten in polacco non si riferiscono alla localizzazione nello spazio degli elementi deittici, bensì alla casa precedentemente menzionata all'interlocutore (questa casa $=$ la casa di cui stai parlando).

b) l'addizione di un elemento che indica la vicinanza all'ascoltatore la traduzione italiana: Questa loro/vostra casa, apparteneva all'altra persona? la traduzione polacca: Ten państwa dom, należał wcześniej do kogoś innego?

Come possiamo osservare, sebbene le traduzioni presentate mantengano il riferimento alla figura del ricevente, cambia il senso dell'intera frase. Per questa ragione, l'addizione di tanti elementi può essere riconosciuta come troppo lontana dalla versione originale.

Come abbiamo notato, in italiano è il verbo venire che prende l'opportunità di segnalare la posizione dell'ascoltatore. Ciò può apportare alcuni problemi nella sua traduzione in spagnolo o in polacco.

(10) Cosa faccio, vengo con Roberta?

Il verbo venire nell'esempio (10) indica che il soggetto svolge il movimento verso la persona dell'ascoltatore. Questa nozione risulta molto difficile da tradurre in spagnolo o in polacco. Di nuovo prendiamo in considerazione due opzioni:

a) l'omissione dell'allusione alla figura dell'ascoltatore

la traduzione spagnola: ¿Qué hago, voy con Roberta?

la traduzione polacca: Co mam zrobić, przyjść $z$ Roberta?

b) l'addizione di un elemento che indica la vicinanza all'ascoltatore

la traduzione spagnola: ¿Qué hago, voy a tu casa con Roberta?

la traduzione polacca: Co mam zrobić, przyjść do ciebie z Roberta?

L'opzione b) permette di sottolineare che il movimento svolgerà verso la persona dell'ascoltatore. Malgrado che le traduzioni nel punto $b$ ) siano più prossime/vicine al siginificato della versione italiana, mi sembrano troppo lunghe e, in conseguenza, poco probabili nel linguaggio parlato.

\subsection{I tratti [dinamico] e [statico]}

Alcune unità deittiche non sono capaci di segnalare la localizzazione di un'entità, ma anche di mostrare una percezione dinamica o statica della entità in questione. Tale è il caso dell'opposizione degli avverbi spagnoli aquí/acá e allí/allá (Brzozowska-Zburzyńska 2005: 67-68). Aquí e allí segnalano i posti considerati come statici, mentre acá e allá designano i punti finali di una traiettoria. Siccome né gli avverbi italiani né quelli polacchi svolgono questa funzione, la questione può risultare complicata nel caso della traduzione. Vediamo il seguente esempio:

(11) y me alegro muchísimo de que los comercios se hayan venido todos para acá 
Nell'esempio (11) il dimostrativo acá suscita due interpretazioni: il negozio adesso si trova nello spazio del parlante e questa situazione è frutto di un movimento, di uno spostamento. Le possibili traduzioni sono capaci di dimostrare la prima nozione, ma non la seconda.

La traduzione italiana: E sono contenta che tutti i negozi si siano venuti qui.

La traduzione polacca: I cieszę się, że cały handel przenióst się tutaj.

Né qui, né tutaj presentano un valore di movimento. Inoltre, non esiste alcun elemento linguistico che manifesti/esprimi questa nozione. D'altra parte, dobbiamo rifletterci se è imprescindibile sottolineare il valore di movimento dell'avverbio dimostrativo. Nell'enunciato spagnolo ci sono due unità che indicano lo spostamento: il verbo venir e l'avverbio acá. Nella versione italiana e nella polca si trova solamente una: l'avverbio qui o tutaj. Ciò nonostante, entrambe le traduzioni trasmettono un significato molto simile a quello dell'enunciato spagnolo. Forse per diffondere la stessa informazione, lo spagnolo ha bisogno di manifestarla tramite due categorie (il verbo e l'avverbio), mentre l'italiano e il polacco solamente tramite una sola (il verbo).

\subsection{La problematica dei verbi polacchi}

Come dimostra la tabella della classificazione dei verbi deittici, in italiano e in spagnolo la divisione tra questi verbi è vincolata alla posizione delle persone (non) presenti nella conversazione. Nel caso del polacco, la questione è più complicata. La scelta del verbo dipende dalla prospettiva che si assume. Ogni verbo può apparire con tutte le persone a seconda dell'elemento che si vuole sottolineare. Per questo, tutti i seguenti esempi sono possibili:

(12) Przychodzisz do mnie./Przychodzę do ciebie./Przychodzi do mnie.

(13) Idziesz do mnie./Idę do ciebie./Idzie do mnie.

La traduzione in polacco degli enunciati italiani o spagnoli che contengono i verbi deittici dipende dalla prospettiva che si seleziona. Esistono i casi in cui, per il contesto, è facile scegliere la forma più adeguata:

(14) Prendo il tram, vengo a scuola per le otto.

Il significato della frase intera ci indica che la traduzione in polacco sarebbe: Jadę tramwajem, przyjdę do szkoły o ósmej.

Ciò nonostante, a volte ci sono due possibilità delle traduzioni:

(15) - Ma me lo porti tu qua?

- Sì sì, vengo io lì. 
La traduzione 1: Ale przyniesiesz mi to tutaj? Tak, tak, idę do ciebie.

La traduzione 2: Ale przyniesiesz mi to tutaj? Tak, tak, przyjdę do ciebie.

Nell'esempio (15) il verbo venire indica il movimento verso l'ascoltatore. Per questo, aggiungiamo l'espressione do ciebie. Per tradurre l'azione di movimento possiamo usare il verbo idę $\left(\mathrm{n}^{\circ} 1\right)$, così sottolineiamo il movimento, o przyjdę $\left(\mathrm{n}^{\mathrm{o}} 2\right)$ e così enfatizziamo l'arrivo al punto di destino.

\section{Conclusione}

Come abbiamo cercato di mostrare tramite questa breve analisi, ogni lingua presenta il proprio metodo di codificare i rapporti spazio-temporali. Sebbene le lingue in questione, particolarmente l'italiano e lo spagnolo, si caratterizzino per i simili sistemi deittici, traducendo alcuni degli enunciati possiamo trovare un'ampia gamma dei problemi che ostacolano la scelta di un corrispondente appropriato. Ciò nonostante, vorrei sottolineare il fatto che le lingue codificano le stesse nozioni sebbene a volte lo facciano tramite le categorie diverse.

Ci sono dei valori che in questo mondo, così differenziato, si presentano uguali. Una di queste nozioni è lo spazio. In tutte le regioni i parlanti esprimono, nell'uno o nell'altro modo, che le entità si trovano vicino o lontano da loro oppure verso dove si dirige il loro movimento. L'unica cosa della quale dobbiamo renderci conto è che per trasmettere questa nozione dello spazio si utilizzano le categorie distinte secondo un determinato sistema linguistico.

\section{Bibliografia}

Brzozowska-Zburzyńska B., 2005, Los marcadores de la deíxis espacial en espańol y en francés, [in:] Estudios de lingüística, 19, pp. 65-84.

Brzozowska-Zburzyńska B., 2012, La expresión lingüística de las relaciones espaciales en espańol, [in:] La construcción y el funcionamiento del espacio en literatura y lingüística, M. Falska, B. Brzozowska-Zburzyńska (ed.), Wydawnicwto UMCS, Lublin, pp. 93-109.

Bühler K., [1934] 2004, Teoria języka, trad. J. Koźbiał, Universitas, Kraków.

Carbonero C.P., 1979, Deíxis especial y temporal en el sistema lingüístico, Publicaciones de la Universidad de Sevilla, Sevilla.

Coseriu E., 1989, Teoría del lenguaje y lingüística general, GREDOS, Madrid.

Fillmore Ch., 1997, Lectures on Deixis, Center for the Study of Language and Information, Stanford. 
Ledgeway A., 2015, Varieties in Italy, [in:] Manuals od Romance Linguistics. Volume 6. Manual of Deixis in Romance Languages, Günter Holtus, Fernando Sánchez Miret, De Gruyter, Berlin, pp. 63-101.

Moreno Cabrera J.C., 1987, Fundamentos de Sintaxis General, SINTESIS, Madrid.

Ruiz A.J.C., 1998, Curso de tipología lingüística. Enfoque funcional, Publicacions de la Universitat Jaume I, Castellón.

Vanelli L., Renzi L., 1995, La deissi, [in:] Grande grammatica italiana di consultazione, Vol. 3, L. Renzi, G. Salvi, A. Cardinaletti, Il Mulino, Bologna, pp. 261-375.

Vicente M.J.A., 1994, La deíxis. Egocentrismo y subjetividad en el lenguaje, Universidad de Murcia, Murcia.

Ewa Urbaniak

\section{The translation of the deictic expressions}

Summary

The deictic expressions are linguistic units that indicate the localization of an object in time and space. Every language is characterized by different deictic structures that depend on various linguistic and extralinguistic factors. The analysis of the factors that determine the deictic systems in Italian, Spanish and Polish demonstrates various problems associated with the translation of that kind of structures and offers different ways of solving them.

Keywords: deixis; space; time; demonstrative adjectives; demonstrative adverbs; deictic verbs. 


\section{La traduzione delle espressioni deittiche}

Riassunto

Le espressioni deittiche sono tutte le unità linguistiche che indicano la localizzazione di un oggetto nello spazio e nel tempo. Ogni lingua si caratterizza per l'altra struttura deittica dipendente da vari fattori linguistici ed extralinguistici. L'analisi dei fattori che condizionano i sistemi deittici dell'italiano, dello spagnolo e del polacco dimostra vari problemi legati alla traduzione di questo tipo di costruzioni linguistiche e propone distinti modi di risolverli.

Parole chiave: deissi; spazio; tempo; aggettivi dimostrativi; avverbi dimostrativi; verbi deittici. 